\title{
Input Shaping for Sway Control in Gantry Cranes
}

\author{
Jeslin Thalapil ${ }^{1}$ \\ ${ }^{1}$ Department of Mechanical Engineering, Indian Institute of Technology, Bombay, Mumbai-400076, India
}

\begin{abstract}
Bridge Cranes or Gantry Cranes are an essential element in the day to day activities of a manufacturing plant. The most common operation of a gantry crane is point to point transportation of payload. The use of cables for hoisting and support of payload can lead to natural swaying. This natural sway is detrimental to safe and efficient operation. Swinging degrades the speed, accuracy, and safety of transport operations. It lowers the speed of transport operations because the payload swing must die out before the payload can be safely lowered into position. The swing makes it difficult to perform alignment, fine positioning, or other accuracy driven tasks. Swing also causes safety problems because of the potential for collisions with objects or people. Under certain conditions, the problem gets more complicated when the payloads creates a double pendulum effect. This report develops a technique for improving the control of two mode double pendulum crane called input shaping. Input shaping is a control strategy that uses a series of impulses to modify the reference command to suppress unwanted vibration in a system. This report presents a method for optimizing a robust input shaper called Specified insensitivity input shapers using the knowledge of amplitude contributions of each mode to the overall response.
\end{abstract}

Keywords Command Shaping, Input Shaping, Gantry Cranes, Sway Control

\section{Introduction}

Cranes have been used for thousands of years to lift and transport heavy materials. They are used in shipyards, construction sites, and warehouses. In all cases the most common operation of a crane is point-topoint transport of a payload. The most common types of cranes used are Bridge cranes and Tower Cranes. Figure 1 shows the most commonly used bridge as well as tower crane.

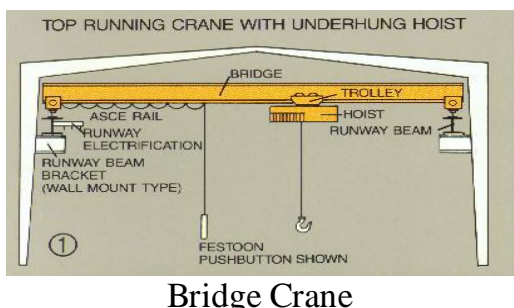

Bridge Crane

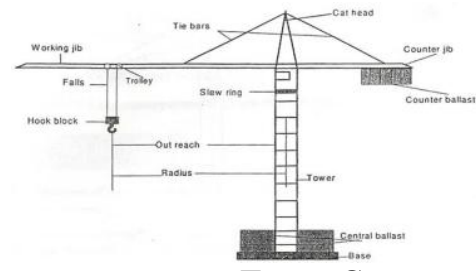

Tower Crane

Figure 1.1. Types of cranes

There are three factors are of paramount importance in crane operations: speed, accuracy, and safety. All cranes use cables to hoist and support the payload. And because of this structure, the payload swings during handling operations. The natural sway of crane payloads degrades speed, accuracy and safety.

If the crane behaves like a single pendulum, an experienced human operator can act as the feedback controller. He can eliminate much of the payload sway by causing an oscillation during deceleration that cancels the oscillation induced during acceleration. However, in most cases certain types of payloads and riggings induce double pendulum dynamics. Under these conditions, the manual method of eliminating oscillation becomes difficult, even for skilled slingers.

The most widely used control method for reducing payload vibration is to simply limit the acceleration and maximum velocity. When the crane operator pushes the button, the crane motors are slowly ramped up to the full speed. The resulting effect is command smoothing that acts as a low pass filter. This method is somewhat effective, but it leads to a sluggish response and large deceleration distances. This method increases the travel time from point to point payload handling and hence is not preferred. If a computer controller is utilized and cable swing is considered in the control design, the time optimal commands that result in zeroresidual vibration can be generated. The implementation is often impractical because the boundary condition i.e. the point to point distance (the move length) must be known at the outset. Another method for controlling sway is the technique of feedback control. Using feedback control is challenging due to the difficulty of measuring the payload motion. The crane would require a camera mounted on the trolley to measure the payload swing in the horizontal plane and velocity and position sensors. 


\subsection{Input Shaping}

Another successful approach uses the technique of Input Shaping to reduce the crane sway. Input shaping is easier to derive and implement than time-optimal control schemes and does not require feedback mechanisms of closed-loop and adaptive controllers. Input Shaping is implemented in real time by convolving the command signal with an impulse sequence (an input shaper). The result of the convolution is then used to drive the crane motors. Real Time convolution only requires a few multiplication and addition operations at each time step. Therefore, it can be implemented on the simplest of digital processors.

The earliest incarnation of input shaping was developed in 1950 by Smith. His posicast control ensures there will be zero residual vibration when the system model is perfect, and hence it is referred to as a zero vibration (ZV) shaping.

This input shaping process is demonstrated in figure 1 using a typical velocity pulse and an input shaper consisting of three impulses.

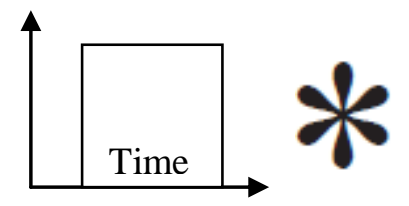

0

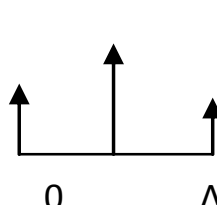

$\Delta$

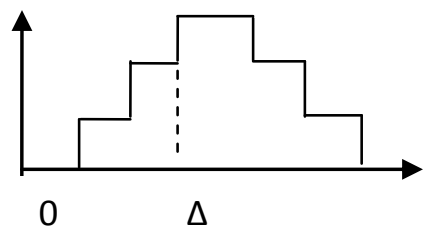

0

Figure 1.2: Input Shaping of a pulse input

The velocity pulse commands are very common in crane control because human operators often press on-off buttons to drive cranes. Proper timing and scaling of the impulses can ensure that the payload oscillation is suppressed to within a desired tolerance

\section{Modelling Of Gantry Cranes}

In order to apply multiple-mode vibration suppression using input shapers to cranes, the dynamics of the crane must be understood. This chapter deals with the dynamics of crane that act as a double pendulum with point mass payload as well as the dynamics of a distributed payload mass.

2.1. Double Pendulum Dynamics

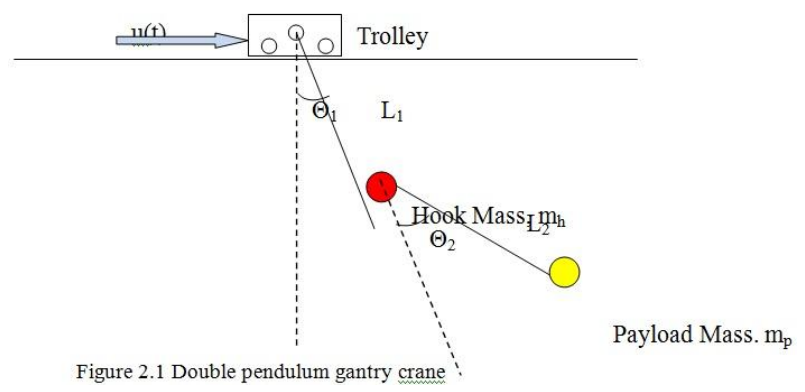

Figure 2.1 shows a schematic representation of a double pendulum gantry crane. The crane is moved by a applying force, $\mathrm{u}(\mathrm{t})$, to the trolley. A cable of length $\mathrm{L}_{1}$, hangs below the trolley and supports a hook of mass, $m_{h}$, to which a payload is attached using riggings cables. Hence the rigging and payload can be modeled as a second cable of length $L_{2}$, and a point mass, $m_{p}$.

Assuming that the cable and rigging lengths do not change, the linearized equations of motion, assuming zero initial conditions, are:

$$
\begin{aligned}
& \theta_{1}^{\prime}(t)=-\left(\frac{g}{L_{1}}\right) \theta_{1}+\left(\frac{g}{L_{1}} R\right) \theta_{2}-\frac{u(t)}{L_{1}} \\
& \theta_{2}^{*}(t)=\left(\frac{g}{L_{1}}\right) \theta_{1}-\left(\frac{g}{L_{2}}+\frac{g R}{L_{2}}+\frac{g R}{L_{1}}\right) \theta_{2}+\frac{u(t)}{L_{1}}
\end{aligned}
$$

Where $\theta_{1}, \theta_{2}$ are the two pendulum angles, $\mathrm{R}$ is the ratio of payload mass to the hook mass $\left(\frac{m_{2}}{m_{1}}\right)$.

The linearized frequencies [2] of the double pendulum are

$$
\omega_{1,2}=\sqrt{\frac{g}{2}} \sqrt{(1+R)\left(\frac{1}{L_{1}}+\frac{1}{L_{2}}\right) \mp \beta}
$$

Where

$$
\beta=\sqrt{(1+R)^{2}\left(\frac{1}{L_{1}}+\frac{1}{L_{2}}\right)^{2}-4\left(\frac{1+R}{L_{1} L_{2}}\right)}
$$


The frequencies depend on the two cable lengths and the mass ratio.

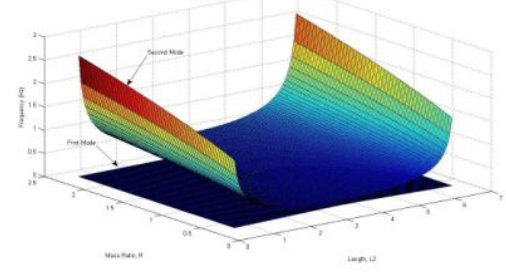

Figure 2.2 Variation of low and high frequencies

Figure 2.2 shows the two oscillation frequencies as a function of mass ratio and rigging length, $\mathrm{L}_{2}$, (assumption that the sum of lengths $\mathrm{L}_{1}+\mathrm{L}_{2}$ remains a constant). The low frequency changes very little and corresponds closely to the frequency of a single pendulum. On the other hand, the second mode has a strong dependence on the rigging length.

The low frequency is maximized when the two cable lengths are equal. But the variation is very little from the median value. In contrast, the second mode deviates highly over the same parameter range. The low frequency changes very little and corresponds closely to the frequency of a single pendulum $\left(\mathrm{L}_{1}+\mathrm{L}_{2}\right)$.

The responses of two swing angles, $\theta_{1}, \theta_{2}$, to an impulse of magnitude $\mathrm{A}$, introduced at time $\mathrm{t}_{0}$, are:

$$
\begin{gathered}
\theta_{1}(t)=\frac{A \omega_{1}\left(1+\omega_{2}^{2} L_{1} a\right)}{k} \sin \left\{\omega_{1}\left(t-t_{0}\right)\right\}-\frac{A \omega_{2}\left(1+\omega_{1}^{2} L_{1} a\right)}{k} \sin \left\{\omega_{2}\left(t-t_{0}\right)\right\} \\
\theta_{2}(t)=\frac{A \omega_{1}}{k} \sin \left\{\omega_{1}\left(t-t_{0}\right)\right\}-\frac{A \omega_{2}}{k} \sin \left\{\omega_{2}\left(t-t_{0}\right)\right\}
\end{gathered}
$$

Where

$$
\begin{aligned}
& \alpha=\frac{-g(1+R)}{\omega_{1}^{2} \omega_{2}^{2} L_{1} L_{2}}, \text { and } \\
& k=\beta L_{1} g
\end{aligned}
$$

If we assume small angles, then the impulse response of the payload in the horizontal direction can be approximated as

Where

$$
x(t)=C_{1} \sin \left(\omega_{1} t+\varphi_{1}\right)+C_{2} \sin \left(\omega_{2} t+\varphi_{2}\right)
$$

$$
\begin{aligned}
& C_{1}=\frac{\omega_{1} L_{1}\left(1+\omega_{2}^{2} \alpha\left(L_{1}+L_{2}\right)\right)}{k} \sqrt{\left(\sum_{j=1}^{n} A_{j} \cos \left(\omega_{1} t_{j}\right)\right)^{2}+\left(\sum_{j=1}^{n} A_{j} \sin \left(\omega_{1} t_{j}\right)\right)^{2}} \\
& C_{2}=\frac{-\omega_{2} L_{1}\left(1+\omega_{1}^{2} \alpha\left(L_{1}+L_{2}\right)\right)}{k} \sqrt{\left(\sum_{j=1}^{n} A_{j} \cos \left(\omega_{2} t_{j}\right)\right)^{2}+\left(\sum_{j=1}^{n} A_{j} \sin \left(\omega_{2} t_{j}\right)\right)^{2}}
\end{aligned}
$$

The coefficients $\mathrm{C}_{1}$ and $\mathrm{C}_{2}$ indicate the contributions of each mode to the overall payload response.

The design of input shaper should be such that the double pendulum crane moves with very little residual vibration, hence, we need to limit the maximum amplitude of the residual vibration from a series of impulses. The maximum amplitude can be found out by adding the maximum amplitude due to each mode.

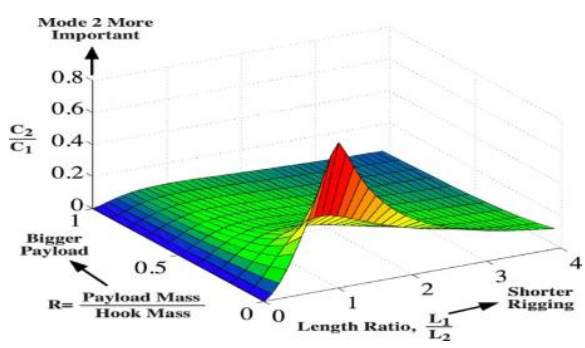

Figure 2.3. Ratio of high mode amplitude to low mode amplitude

Figure 2.3 shows the ratio of the mode 2 to mode 1 for a range of length ratio and mass ratios [2]. It is clear that the input shaping would be essential for systems where the payload to hook mass ratio is less, and when the hook cable length and rigging lengths are approximately equal. 


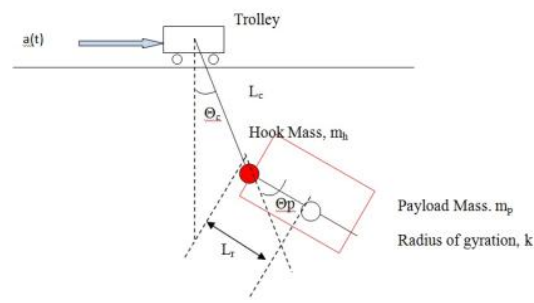

Figure 2.4 Distributed mass gantry crane system

Figure 2.4 shows a schematic representation of a planar bridge crane with a distributed payload. The acceleration of the overhead trolley is a(t). A mass less cable, of length $\mathrm{L}_{\mathrm{c}}$, hangs below the trolley s a hook, of mass $m_{h}$, to which the payload is attached. The distance from the hook to the centre of mass of the payload is $L_{r}$. The payload is characterized by its mass $\mathrm{m}_{\mathrm{p}}$ and radius of gyration " about its centre of mass.

If the cable and rigging lengths do not change, then the linearized equations of motion [1] are

$\left(m_{p} k^{2}+L_{s} m_{p}\left(L_{r}+L_{p}\right)\right) \theta_{p}+\left\{m_{p} k^{2}+m_{h} L_{c}^{2}+m_{p}\left(L_{c}^{2}+L_{r}^{2}+2 L_{c} L_{r}\right)\right\} \theta_{c}=-\left(g L_{c} m_{h}+g m_{p} L_{c}+\right.$ $\left.g m_{p} L_{r}\right) \theta_{c}-\left(g m_{p} L_{r}\right) \theta_{p}-\left\{L_{c} m_{n_{1}}+m_{p}\left(L_{c}+L_{r}\right)\right\} a(t)$

$\left(m_{p} k^{2}+m_{p} L_{r}^{2}\right) \theta_{p}+\left\{m_{p} k^{2}+L_{r} m_{p}\left(L_{r}+L_{c}\right)\right\} \theta_{c}=-L_{r} m_{p} g \theta_{c}-L_{r} m_{p} g \theta_{p}-L_{r} m_{p} a(t)$

Where $\theta_{c}, \theta_{p}$ are the swing angles that measure the cable rotation and payload rotation.

The Linearized frequencies [1] of the system are:

$$
\begin{gathered}
\omega_{1}^{2}=\frac{g(1+R)}{2 L_{c}}(\alpha-\beta) \\
\omega_{2}^{2}=\frac{g(1+R)}{2 L_{c}}(\alpha+\beta)
\end{gathered}
$$

where

$\alpha=\frac{k^{2}+L_{r} L_{c}+L_{r}^{2}}{L_{F}^{2}+k^{2}(R+1)}$
$\beta=\sqrt{\alpha^{2}-\frac{4 L_{r} L_{c}}{(R+1)\left\{L_{\gamma}^{2}+k^{2}(1+R)\right\}^{2}}}$, where $\mathrm{R}$ is the mass ratio.

The frequencies depend on the two cable lengths, the radius of gyration of the payload, $\boldsymbol{k}$ and the mass ratio, R.

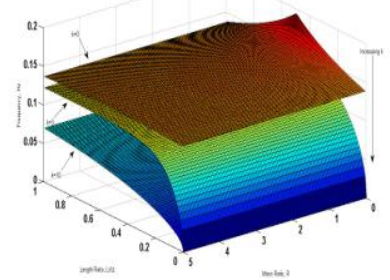

Figure 2.5 Low Mode frequency

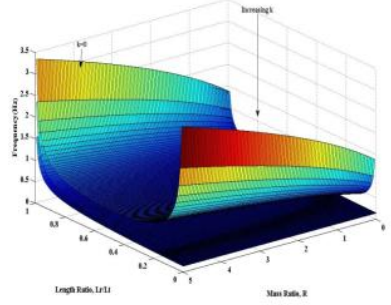

Figure 2.6.High Mode frequency

The figure 2.5 and 2.6 shows the variation of low frequency and high frequency modes variation with length ratio, mass ratio and radius of gyration. Table 1 shows the median value of the two frequencies and how they vary with radius of gyration. The low mode frequency doesn't vary much from the median value when the radius of gyration is near to 1 .

\begin{tabular}{|c|c|c|c|}
\hline \multirow{2}{*}{ Radius of Gyration $\mathbf{k}$} & Mode & $\begin{array}{c}\text { Median } \\
\text { Frequencies(Hz) }\end{array}$ & Variation \\
\hline $\mathbf{0}$ & Low & 0.14 & $11 \%$ \\
& High & 0.572 & $78 \%$ \\
\hline $\mathbf{1}$ & Low & 0.1388 & $12 \%$ \\
& High & 0.422 & $84 \%$ \\
\hline $\mathbf{5}$ & Low & 0.0963 & $56 \%$ \\
& High & 0.22 & $77 \%$ \\
\hline $\mathbf{1 0}$ & Low & 0.0552 & $65 \%$ \\
& High & 0.1988 & $73 \%$ \\
\hline $\mathbf{5 0}$ & Low & 0.0155 & $68 \%$ \\
& High & 0.1936 & $69 \%$ \\
\hline
\end{tabular}

Table 1: Frequency Ranges for a variety of payloads 
The analysis indicates that an oscillation control scheme would need more robustness to variations in the second mode than in the first mode. However, if the amplitude of the second mode is very small, then the controller need not attend to the second mode. This can be examined by decomposing the overall dynamic response into components from the low and high frequencies.

The payload response from an impulse input A [1] can be expressed as

$x(t)=C_{1} \sin \left(\omega_{1} t\right)-C_{2} \sin \left(\omega_{2} t\right)$

where

$C_{1}=A \frac{L_{c}+\omega_{2}^{2} L_{y} \gamma\left(L_{c}+L_{y}\right)}{g \beta(R+1)} \omega_{1}$

$C_{2}=A \frac{L_{c}+\omega_{1}^{2} L_{r} \gamma\left(L_{c}+L_{r}\right)}{g \beta(R+1)} \omega_{2}$

where

$\gamma=\frac{-g(R+1)}{\omega_{1}^{2} \omega_{2}^{2}\left(L_{r}^{2}+k^{2}(R+1)\right)}$

The coefficients $\mathrm{C}_{1}$ and $\mathrm{C}_{2}$ give the amplitude contribution of each mode to the overall impulse response.

The maximum amplitude is thus

$$
V_{\max }=\left|C_{1}\right|+\left|C_{2}\right|
$$

The variation of second mode amplitude contribution with mass ratio, length ratio and radius of gyration is shown in figure 2.7 [1]. The figure reveals that double pendulum effects are most important for point mass payloads $(\mathrm{k}=0)$ with low payload to hook mass ratio and length ratios near 0.5 .

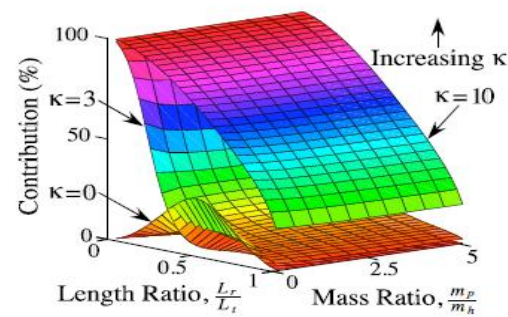

Figure 2.7.Amplitude contribution of $\omega_{2}$

It's clear from the above plot that the double pendulum effects are most important for point mass payloads $(\mathrm{k}=0)$ with low payload to hook mass ratio and length ratios of 0.5 . The dynamics of the distributed payload crane are dependent on the payload mass, rigging length and radius of gyration of the payload. The payload mass has significant effect on the system frequencies and amplitude contributions when the mass of the payload is small relative to the mass of the hook and radius of gyration is small. The radius of gyration has a large effect on the frequencies and the amplitude contributions when it is small but becomes less dominant as it increases. The rigging length has the largest influence on both the frequencies and the amplitude contributions when the radius of gyration grows large.

\section{Input Shaping For Double Pendulum Gantry Cranes}

\subsection{Principle of Input Shaping}

Input shaping command, which can be considered as a feed forward control, is an algorithm that shapes the input command to reduce the residual vibration of the system. Input shaping limits the residual vibration by generating a command profile that tends to cancel its own vibration. That is, the vibration induced in the first part of the command is cancelled by vibration induced by a later portion of the command. Input shaping is easier to derive and implement than time optimal control schemes and does not require the feedback mechanisms of closed loop and adaptive controllers.

Using estimates of system natural frequencies and damping ratios, a series of impulses, called the input shaper, is designed. Input shaping is obtained in real time by convolving the command signal with a sequence of impulses (input shaper). The result of this convolution is used to drive the system. Thus the input shaper modifies the desired velocity command before it is fed to the crane drives.

A typical implementation of a input shaper on a crane system is shown in figure 3.1. 


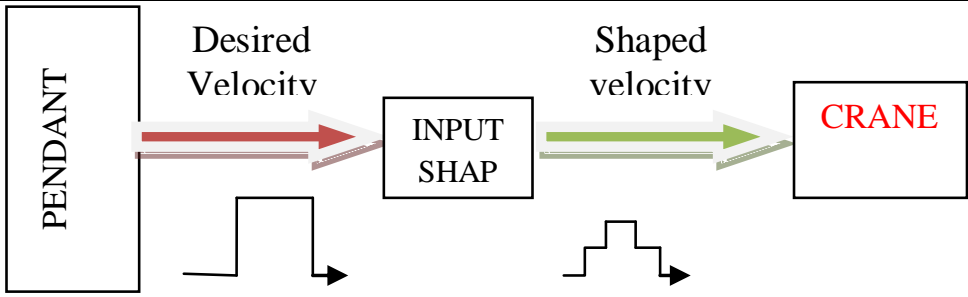

Figure 3.1- Block Diagram of an input shaped crane system

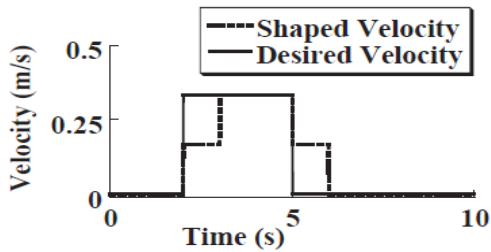

Figure 3.2- Desired and Shaped Velocity Commands

Figure 3.2 shows the original desired command and the new velocity command created by the input shaper. The desired command to achieve the point to point motion is a velocity pulse. In contrast, the shaped velocity command stays at half speed for a short time before reaching the final full on velocity. It is this modification, which the input shaper imparts on the velocity command that causes the payload to move without swinging.

This modification is achieved by applying a convolution filter to the command. The input/output formula for a convolution filter is:

$$
Y(t)=G_{I S}(t) * X(t)
$$

Where $\mathrm{X}(\mathrm{t})$ is the input, $\mathrm{G}_{\mathrm{IS}}(\mathrm{t})$ is the input shaping filtering sequence, $\mathrm{Y}(\mathrm{t})$ is the output, and '*' is shorthand for the convolution integral.

A generalized input shaper is shown in Figure 3.3
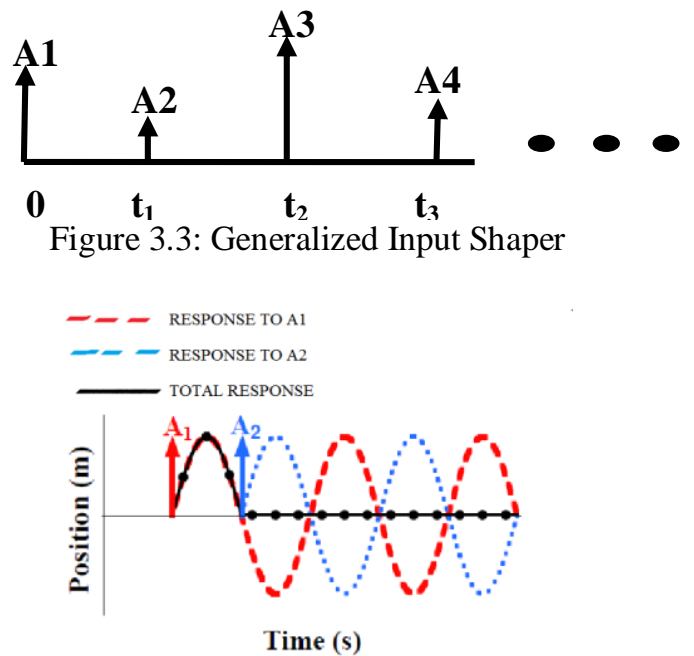

Figure 3.4: Superposition of Impulse responses.( taking only A1 and A2)

The goal is to find out the impulse times and the amplitudes such that the total response has zero residual vibration The residual vibration can be rejected by superposition of two responses and this leads to a shorter settling time. In general, input shaping is based on the linear system theory and is defined as [8];

$$
\begin{aligned}
& y(t)=A \frac{\omega}{\sqrt{1-\zeta^{2}}} e^{-\zeta \omega\left(t-t_{0}\right)} \sin \left\{\omega \sqrt{1-\zeta^{2}\left(t-t_{0}\right)}\right\} \\
& \text { Impulse Magnitude, } K=e^{\frac{\zeta x}{\sqrt{1-\zeta^{2}}}} \\
& \text { Impulse Delay, } \Delta T=\frac{\pi}{\omega \sqrt{1-\zeta^{2}}}
\end{aligned}
$$

Where $\mathrm{A}$ is the amplitude of vibration, $\omega$ is the natural frequency and $\zeta$ is the damping ratio, $t$ is the time and $t_{0}$ is the time of impulse applied to the system. Hence, a simple input shaper with two impulses is designed from the natural frequency and damping ratio. 
To obtain exactly zero residual vibration is practically impossible on real machines, and hence, the input shaper should be designed to yield non-zero, but low levels of sway. The impulse time and amplitudes will be a function of the system natural frequency and damping ratio.

The Laplace transform of equation 2.1 is

$Y(\omega)=G_{I S}(\omega) X(\omega)$

We have chosen the input shaper such that it excites zero vibration at the system's natural frequency or $G_{I S}\left(\omega_{n}\right)=0$. And hence it follows that $Y\left(\omega_{n}\right)=0$. In other words, the shaped input, $\mathrm{Y}(\mathrm{t})$, always has zero frequency content at the system's natural frequency regardless of the input $X(t)$.

Input shaping is a form of Finite impulse Response (FIR) filtering that places zeroes near the locations of the flexible poles of the original system. If $G_{I S}(\omega)$ has zeroes at the poles of $\mathrm{Y}(\omega)$, then the pole-zero cancellation will occur.

\subsection{General Input Shaping Constraints}

In order to determine the impulse amplitudes and time locations of an input shaper, the designer must ensure that they satisfy certain design constraints. The primary design constraint is a limit on the amplitude of vibration caused. We can use the expression for residual vibration, $V_{\max }=\left|C_{1}\right|+\left|C_{2}\right|$, as a constraint equation by requiring $\mathrm{V}_{\max }$ to be less than the tolerable vibration $\mathrm{V}_{\text {tol }}$. The tolerable vibration level comes from the crane and its typical manipulation tasks.

A Mathematical constraint that ensures the vibration with input shaping will be below the tolerable level is $V_{\text {tol }} \geq V_{\text {max }}=C_{1}+C_{2}$

The vibration caused by an input shaper can be limited by the above equation, but if the impulse amplitudes are not constrained, then their values can become very large. This can lead to actuator saturation. The vibration amplitude of an under-damped, second order system from a sequence of $n$-impulses is:

$A_{\Sigma}=\frac{\omega}{\sqrt{1-\zeta^{2}}} e^{-\zeta \omega t_{n}} \sqrt{\left\{\sum_{i=1}^{n} A_{i} e^{\zeta \omega t_{i}} \cos \left(\omega t_{i} \sqrt{1-\zeta^{2}}\right)\right\}^{2}+\left\{\sum_{i=1}^{n} A_{i} e^{\zeta \omega t_{i}} \sin \left(\omega t_{i} \sqrt{1-\zeta^{2}}\right)\right\}^{2}}$

Where $\omega$ is the natural frequency of the system and $\zeta$ is the damping ratio, and $A_{i}$ and $t_{i}$ are the $i^{\text {th }}$ - impulse amplitude and time, respectively. To form non-dimensional vibration amplitude, the equation is divided by the amplitude of residual vibration from a single impulse of unity magnitude at time zero. The resulting expression gives the ratio of vibration with input shaping to that without input shaping. The amplitude of residual vibration from a single unity-magnitude impulse applied at time zero is

$A_{\uparrow}=\frac{\omega}{\sqrt{1-\zeta^{2}}}$

Dividing equation by yields the percentage residual vibration (PRV):

$P R V=V(\omega, \zeta)=e^{-\zeta \omega t_{n}} \sqrt{[C(\omega, \zeta)]^{2}+[S(\omega, \zeta)]^{2}}$

Where

$C(\omega, \zeta)=\sum_{i=1}^{n} A_{i} \ell^{\ell \omega t_{i}} \cos \left\{\omega t_{i} \sqrt{1-\zeta^{2}}\right\}$

$S(\omega, \zeta)=\sum_{i=1}^{n} A_{i} e^{\zeta \omega t_{i}} \sin \left\{\omega t_{i} \sqrt{1-\zeta^{2}}\right\}$

A constraint on residual vibration amplitude can be obtained by $V(\omega, \zeta) \leq V_{\text {tol }}$.

\subsection{Robust Input Shapers}

Input shapers have characteristics that make them easy and effective solutions for eliminating vibration in cranes. Input shaping will eliminate vibration irrespective of the velocity command. Different types of input shapers can be used in the convolution filter. Figure 3.5 shows three basic types of shaper along with the resulting shaped pulse command 


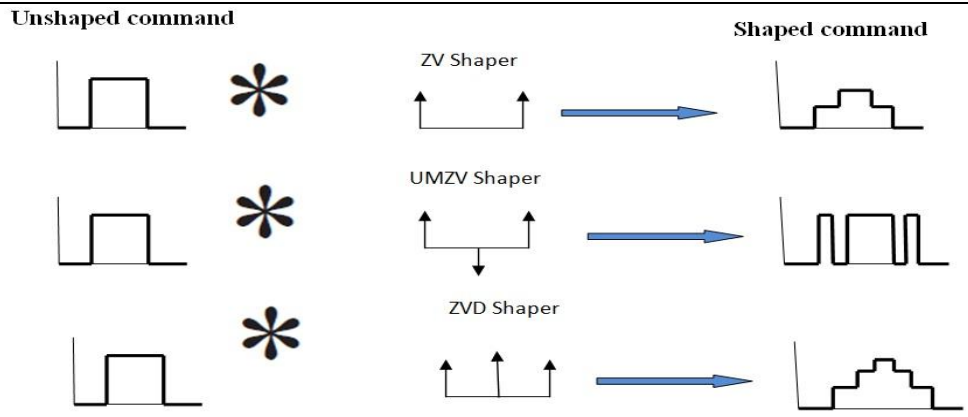

Figure 3.5 Various pulse shaped commands

The Zero Vibration (ZV) input shaper and Derivative (ZVD) are used commonly to suppress the vibration of system in a small and medium range around the design frequency. ZV and ZVD shapers have poor robustness to modeling errors [7]. If the design frequency changes by more than the shaper's inherent insensitivity, then the input shaper was ineffective to suppress the vibration. The robustness of a shaper can be measured using a sensitivity plot, which is a plot of the percentage residual vibration versus the normalized frequency (the actual frequency, $\omega_{\mathrm{a}}$, divided by the modeling frequency, $\omega_{\mathrm{m}}$ ). To compare robustness quantitatively, the insensitivity is defined as the width of the curve that lies below a specified level, normalized by the modeling frequency. The sensitivity plot of ZV and ZVD input shaper [4] is shown in figure 3.6

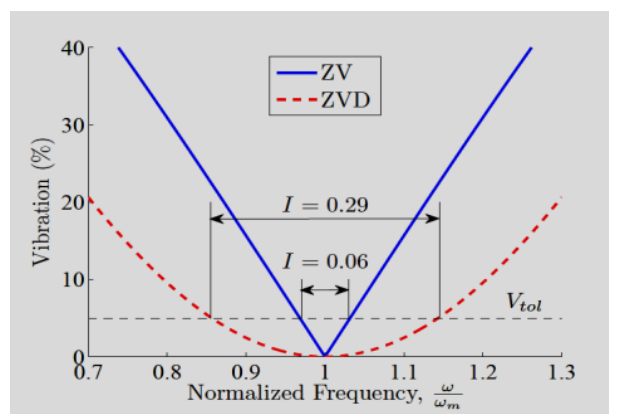

Figure 3.6 Sensitivity plot of ZV and ZVD shapers

One key quantitative measure obtained from a sensitivity curve is the Insensitivity. Insensitivity is the width of the sensitivity curve at a tolerable vibration level, $\mathrm{V}_{\mathrm{tol}}$, with respect to the parameter of interest.

In real applications, the system parameters needed to form an input shaper are not known exactly and hence modeling system parameters to within the tolerances needed for ZV or ZVD shapers is difficult. This led to the development of Robust Input shapers.

The methods used to develop robust input shapers fall loosely into four categories:

$$
\begin{aligned}
& \text { - } \\
& \text { - } \\
& \text { - } \\
& \text { - } \\
& \text { Opthothods ( MIS methods) }
\end{aligned}
$$

Derivative

Tolerable hoc

Numerical

\subsection{Specific Insensitivity input shaper (SI shaper) for gantry cranes}

Specified Insensitivity (SI) input shaping is a robust input-shaping technique that tailors the control robustness to suppress any desired range of frequencies. The Second mode causes the payload oscillation to exceed tolerable levels and hence it must be accounted for when designing the input shaper. A sensitivity curve for an SI shaper is shown in figure 3.7. 


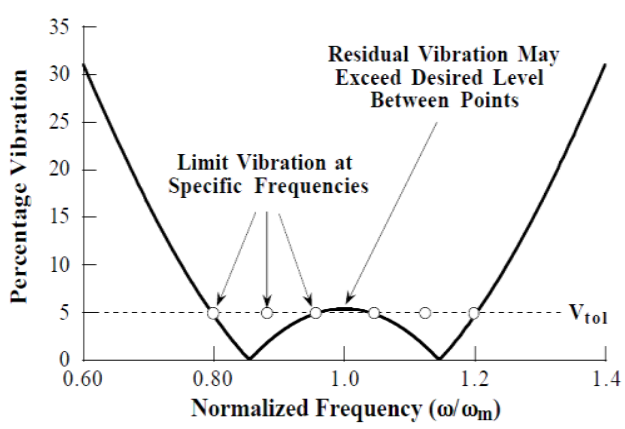

Figure 3.7 Sensitivity curve for SI shaper

Specified Insensitivity shapers provide the greatest level of robustness for an input shaper. The disadvantage is that that an optimization is required to solve for the impulse amplitudes and time locations.

A two mode Specified-insensitivity input shapers are designed by satisfying the constraint equations as listed below:

a) Residual Vibration Constraints: The maximum residual vibration amplitude, $V_{\max }$ should be less than the threshold amplitude $\mathrm{V}_{\text {tol }}$. It has been shown that the robustness can be improved if the vibration is limited to a small value, rather than forced to be exactly zero.

$$
V_{\text {tol }} \geq\left|C_{1}\right|+\left|C_{2}\right|
$$

b) Amplitude Constraints: The vibration caused by an input shaper can be limited by equation (11). However, if the input shaper impulse amplitudes are not considered, then their values can range between positive and negative infinities. There are two possible solutions to this problem: limit the magnitude of the impulse less than a specific value or require all the impulses to have positive values. The shapers discussed here will contain only positive impulses as follows

$$
A_{\mathrm{i}}>0, i=1,2,3 \ldots \ldots, n
$$

Where ' $n$ ' is the number of impulses in the shaper. Impulse amplitudes are also limited to sum to one, which ensures the shaped command reaches the same set point as the unshaped command. The constraint is expressed as:

$$
\sum_{i=1}^{n} A_{i}=1
$$

c) Robustness Constraints: The residual vibration constraint of equation (11) can be used to limit the vibration at a single set of frequencies $\left(\omega_{1}\right.$ and $\left.\omega_{2}\right)$. If the actual crane frequencies coincide with those used in equation (11) to design the shaper, then the oscillation will be eliminated. However, to ensure robustness to modeling errors and parameter variations, the oscillation must remain small over a neighborhood of frequencies that surround the modeling frequencies.

d) Requirement of time optimality: Two mode SI input shaper is designed by minimizing the time of the final input shaper impulse so as to ensure fastest possible solution :

$$
\operatorname{Min}\left(t_{n}\right)
$$

The figure 3.6 shows a basic flow chart for determining an input shaper.

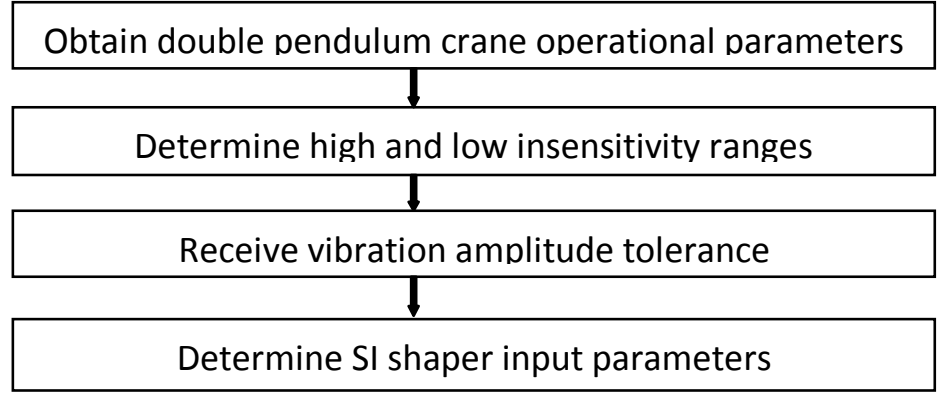

Figure 3.6 Basic flow chart for design of Input shaper

SI input shapers, by design, have a variable insensitivity that can be specified by the designer. The strength of SI shaper, is robustness to modeling errors, but the trade off is an increase in rise time of the system. Hence, from the above four constraints eqn. (11) to (14), a two mode specified insensitivity input shaper is designed. This can be done on any optimization software, like MATLAB optimization toolbar. The operational 
parameters such as cable lengths, payload dimensions, etc. has to be provided by the user. This information can be obtained by crane sensors such as vision systems, load cells, strain gauges, and encoders.

\subsection{Implementation}

The above procedure was implemented in Georgia Tech Institute on a 10 ton bridge crane transporting a long slender beam [1]. This crane has an acceleration of $0.572 \mathrm{~m} / \mathrm{s}^{2}$ and a velocity limit of $0.358 \mathrm{~m} / \mathrm{s}$. The Beam used is $2.5 \mathrm{~m}$ tall, has a diameter of $18 \mathrm{~cm}$ and weighs $22 \mathrm{~kg}$. The hook approximates to $50 \mathrm{~kg}$ point mass. The height of the crane from the floor is $6.2 \mathrm{~m}$. The initial suspension length is $3.5 \mathrm{~m}$ and the shaper designed to suppress oscillations for suspension lengths varying from 2.5 to $3.5 \mathrm{~m}$.

The resulting SI input shaper calculated [1] was

$\left[\begin{array}{c}A_{\mathrm{i}} \\ t_{\mathrm{i}}\end{array}\right]=\left[\begin{array}{cccc}0.1617 & 0.3383 & 0.3383 & 0.1617 \\ 0 & 1.34 & 2.67 & 4.01\end{array}\right]$

Three separate tasks were performed by the crane unshaped as well as with SI input shaper, and the results are tabulated below

\begin{tabular}{|c|c|c|}
\hline Shaper used & $\begin{array}{c}\text { Average } \\
\text { completion time (s) }\end{array}$ & Reduction (\%) \\
\hline Unshaped & 149.0 & -- \\
\hline SI (2 mode) & 22.5 & 84 \\
\hline
\end{tabular}

Table 2: Average completion times moving around an obstacle

\begin{tabular}{|c|c|c|}
\hline Shaper & Button Pushes & Reduction \\
\hline Unshaped & 33 & -- \\
\hline SI (2 mode) & 5 & 85 \\
\hline
\end{tabular}

Table 3: Average operator effort moving around an obstacle

\begin{tabular}{|c|c|c|}
\hline Shaper & $\begin{array}{c}\text { Average completion } \\
\text { time(s) }\end{array}$ & Reduction (\%) \\
\hline Unshaped & 94 & \\
\hline SI (2 Mode) & 37 & 61 \\
\hline
\end{tabular}

Table 4: Average completion times when hoisting over an obstacle

\begin{tabular}{|c|c|c|}
\hline Shaper & Button Pushes & Reduction (\%) \\
\hline Unshaped & 16 & -- \\
\hline SI (2 Mode) & 4 & 75 \\
\hline
\end{tabular}

Table 5: Average operator effort when hoisting over an obstacle

\section{Conclusions}

Input shaping is an effective feed forward control technique that can be implemented on bridge cranes to reduce the payload sway. For certain payloads and rigging configurations, bridge cranes can exhibit double pendulum dynamics. The second mode of natural frequency is significant when the radius of gyration and the rigging length increases. The input shaper has to be designed which can suppress multi mode vibration. Different types of possible input shapers were discussed. The robustness of each of these input shapers is discussed. Furthermore, the input shaper can be made robust to modeling errors and parameter variations by suppressing a range of possible frequencies.

Chapter 1 of this report explained the types of cranes and the need for sway control of gantry cranes in industries. It also discussed the basics of input shaping that can be used to modify the input signal command.

Chapter 2 discusses the dynamics of cranes moving with distributed payloads. Equations for determining natural frequencies and amplitudes were also discussed. It was shown that the frequencies of a distributed payload crane decreases as the radius of gyration increases or the rigging length increases in proportion to the total length of the system. It was also shown that the second mode of the system is more dominant and hence, multi-mode vibration suppression is needed for the same. 
Chapter 3 discusses the various types of input shapers as well as the robustness of these shapers to modeling errors and parameter variations. An effective input shaper, known as specified insensitivity input shaper which is the most robust to modeling errors was also designed. The implementation of SI input shaper was done by the Georgia Tech 10-ton laboratory crane. The effectiveness of SI input shaper to various tasks is also shown.

\section{References}

[1] Manning, R., Clement, J., Kim, D., and Singhose, W., 2010, "Dynamics and Control of Bridge Cranes Transporting DistributedMass Payloads,” ASME J. Dyn. Syst., Meas., Control, 132, p. 014505

[2] Singhose, W., Kim, D., and Kenison, M., 2008, "Input Shaping Control of Double- Pendulum Bridge Crane Oscillations," ASME J. Dyn. Syst., Meas., Control, 130, p. 03450

[3] Blackburn, David, "Command Shaping for Vibration Reduction in Nonlinear Cabled Systems" in Mechanical Engineering Atlanta, GA: Georgia Institute of Technology, 2006

[4] Manning, Raymond, "Improving the control of two-mode flexible systems with input shaping" in Mechanical Engineering Atlanta, GA: Georgia Institute of Technology, 2008

[5] Vaughan, Joshua, "Dynamics and Control of Mobile Cranes" in Mechanical Engineering Atlanta GA: Georgia Institute of Technology

[6] Singer, N. C., and Seering, W. P., 1990, "Preshaping Command Inputs to Reduce System Vibration,” ASME J. Dyn. Syst., Mea s., Control, 112, pp. 76-82.

[7] Chul-Goo Kang, 2011, "Performance Measure of Residual Vibration Control” ASME J. Dyn. Syst., Meas., Control, 133, p 044501

[8] Singer, Neil, 1988, "Residual Vibration Reduction in computer controlled machines", MIT Artificial Intelligence Laboratory. 